sufficient to rule out any nonlinear or asymmetric structure for carbon suboxide. It is disappointing that we cannot make a complete vibrational analysis of the absorption spectrum, but it appears quite probable that absorption studies made under higher resolution will enable the analysis to be completed. We are grateful to Professor Halford for his kindness in furnishing us with the carbon suboxide, to Professor Randall for placing the spectrometer at our disposal and particularly to Professor Dennison for helpful discussions.

\title{
Calculation of the Heat Capacity Curves of Crystalline Benzene and Benzene- $d_{6}$
}

\author{
R. C. LORD, JR.* \\ Department of Chemistry, University of Michigan, Ann Arbor, Michigan \\ J. E. Ahlberg and D. H. Andrews \\ Department of Chemistry, Johns Hopkins University, Baltimore, Maryland
}

(Received March 27, 1937)

\begin{abstract}
The heat capacity curve for solid benzene has been computed with the help of the set of internal frequencies previously proposed by Lord and Andrews. The agreement with experiment is satisfactory over the entire temperature range in which the internal frequencies contribute, indicating that the frequency values are essentially correct. A prediction of the heat capacity of benzene- $d_{6}$ has also been made.
\end{abstract}

$\mathrm{T}$ HE existence of a complete set ${ }^{1}$ of values for the internal frequencies of the benzene molecule makes it possible for the first time to calculate directly the contribution of these degrees of freedom to the heat capacity of crystalline benzene. Earlier attempts ${ }^{2}$ to carry out computations of this sort involved empirical and rather rough estimates of these frequencies, and led only to qualitative information concerning the specific heat of the crystal. A precise calculation of the heat capacity of solid benzene is of interest, particularly in view of the experimental data obtained at "helium" temperatures recently by Ahlberg, Blanchard and Lundberg ${ }^{3}$ and the other excellent values at higher temperatures which can be compared with calculated values. Our discussion will be concerned with this calculation and the conclusions to be drawn from such a comparison.

When thermal energy is absorbed at constant pressure by a crystalline solid such as benzene, this energy is distributed among: (1) the oscilla-

* National Research Council Fellow.

1 Lord and Andrews, J. Phys. Chem. 41, 149 (1937).

2 D. H. Andrews, Proc. Roy. Soc. Amst. 29, 744 (1926); Chem. Rev. 5, 533 (1928); E. O. Salant, Proc. Nat. Acad. Sci. 12, 334, $370(1926)$.

${ }^{3}$ J. E. Ahlberg, E. R. Blanchard and W. O. Lundberg, J. Chem. Phys. 5 , 539 (1937). tions of the crystal lattice; (2) the internal vibrations of the molecules composing the lattice; and (3) the work of expanding the lattice. Before discussing these three components of the specific heat, we must emphasize that our ultimate interest lies in the contribution (2). Contributions (1) and (3) will be evaluated as accurately as possible by means of the traditional expressions for them, but it is not our purpose to improve these expressions or to criticize their theoretical origins.

Experience has shown that the share of the lattice vibrations in the specific heat of a crystal is ordinarily described rather accurately by the familiar Debye function $D(x)$ :

$$
\begin{aligned}
\left.C_{v} \text { (lattice }\right) & =3 R D(x), \\
D(x) & =\frac{12}{x^{3}} \int_{0}^{x} \frac{z^{3} d z}{e^{z}-1}-\frac{3 x}{e^{x}-1},
\end{aligned}
$$

in which $x$ is the parameter $\left(h \nu_{D} / k T\right) . \nu_{D}$ is the well-known Debye characteristic frequency which must be determined in some way if we are to utilize the function $D(x)$.

The physical significance of $\nu_{D}$ has been the subject of much discussion. In a recent critique 
of the Debye theory, Blackman ${ }^{4}$ points out that there is no theoretical justification for identifying $\nu_{D}$ with any of the actual vibrations of the crystal lattice. The assumption that $\nu_{D}$ is a residual ray frequency, for example, appears to be unwarranted despite the closeness of agreement between $\nu_{D}$ and reststrahlen frequencies for a number of crystals. For our purposes, however, the physical meaning of $\nu_{D}$ is of secondary importance. If a value of $\nu_{D}$ can be found by means of which $D(x)$ reproduces the lattice $C_{v}$, our requirements will be satisfied.

Before considering the method of evaluating $\nu_{D}$, it is necessary to take up a question arising from the fact that the lattice of solid benzene is not an atomic lattice. Functions of the Debye type apply to crystal lattices made up of mass points having no moment of inertia. Each mass point possesses only three degrees of freedom and the compressional waves in the three dimensions of the space lattice are the crystalline analog of the three degrees of translational freedom allowed to the particles of a monatomic gas. Doubtless the molecules in a benzene crystal may be fairly considered as mass points insofar as the propagation of compressional waves is concerned. These compressional vibrations should therefore take up thermal energy in the manner described by the Debye function. In addition, however, the benzene molecule possesses three moments of inertia and correspondingly three degrees of rotational freedom. If the component molecules of the benzene lattice were rotating freely and independently of one another, the contribution of these rotational degrees of freedom to the heat capacity would be the same as their contribution to $C_{v}$ of gaseous benzene. The benzene molecules do not rotate freely, of course, and therefore we have to determine what the nature of the rotatory motion is and what its effect on the specific heat will be.

Each molecule in the benzene lattice is situated at an equilibrium position in the field of force resulting from the presence of the neighboring molecules. If one molecule be somewhat displaced from this position by a translation or by rotation through some small angle about its center of gravity, it will tend to return to the equilibrium position. The displacement will, however, affect

\footnotetext{
${ }^{4}$ Blackman, Proc. Roy. Soc. A148, 365, 384 (1935).
}

the neighboring molecules, since the force field acting on each of them is partly due to the displaced molecule. This interaction of molecules undergoing translational displacement leads to the compressional waves mentioned above. Similarly angular displacement results in the production of torsional waves, in which the molecules in the crystal lattice execute rotatory oscillation through small angles. The oscillations of each molecule bear definite phase relationships to the oscillations of the others, and the oscillational waves are propagated in all three dimensions of the space lattice. It is our assumption that these torsional waves contribute to the specific heat in the same manner as the compressional waves, and that this contribution is accordingly described by a Debye function $D(x)$. The maximum frequency $\nu_{D}$ for the torsional waves will of course differ in general from $\nu_{D}$ for the compressional vibrations. The expression for the heat capacity of the benzene lattice will be therefore

$$
C_{v} \text { (lattice) }=3 R\left[D\left(x_{1}\right)+D\left(x_{2}\right)\right] .
$$

To evaluate the two $\nu_{D}$ 's required by Eq. (3), we use the following procedure. At low temperatures, say below $50^{\circ} \mathrm{K}$, the internal vibrations and work of expansion make only trifling contributions to the specific heat of benzene. Consequently, the experimentally observed heat capacity corresponds to that given by Eq. (3). Hence, if we select values for $\nu_{D_{1}}$ and $\nu_{D_{3}}$ which reproduce the heat capacity curve over the range $0^{\circ}-50^{\circ} \mathrm{K}$, we can feel confident that these values are the correct ones for use at higher temperatures.

When the two values of $\nu_{D}$ are not widely different, we may say

$$
D\left(x_{1}\right)+D\left(x_{2}\right)=2 D\left(\frac{x_{1}+x_{2}}{2}\right) .
$$

Or, setting $h \nu_{D_{1}} / k=\theta, h \nu_{D_{2}} / k=\theta_{2}$ and $\left(\theta_{1}+\theta_{2}\right) / 2$ $=\theta$,

$$
D\left(\theta_{1} / T\right)+D\left(\theta_{2} / T\right)=2 D(\theta / T) .
$$

From unpublished calculations by Dr. V. Deitz and Mr. C. F. Squire, extending the former's investigations of crystal lattices ${ }^{5}$ it appears that $\theta_{1}$ (for compressional oscillations) in benzene is about 25 percent smaller than $\theta_{2}$ (for torsional

\footnotetext{
${ }^{6}$ V. Deitz, J. Frank. Inst. 219, 459, 565, 703 (1935).
} 
oscillations). When the $\theta$ 's considered lie in the range $100-200$ and differ by 25 percent, the approximation (5) holds to within experimental error at temperatures above $35^{\circ}-40^{\circ} \mathrm{K}$. Even at temperatures considerably lower the approximation is valid to better than 5 percent. Accordingly we may obtain from the heat capacity data at low temperature the value of a single $\theta$ and in terms of this $\theta$ express the heat capacity due to the lattice of crystalline benzene over the entire temperature range of the solid. The equation will be :

$$
C_{v}(\text { lattice })=6 R D(\theta / T) .
$$

The question of the accuracy of Eq. (6) and of possible variatons in $\theta$ will be considered later.

The contribution made by the internal frequencies in the benzene molecule to the specific heat of the crystal is readily calculable. The heat capacity arising from one degree of internal freedom of frequency $\nu_{i}$ is

$$
C_{v}=R E\left(x_{i}\right),
$$

where $x_{i}=h v_{i} / k T$, and the Einstein function $E(x)=x^{2} e^{x} /\left(e^{x}-1\right)^{2}$. Hence the total internal heat capacity will be

$$
C_{v}(\text { internal })=R \cdot \sum_{i=1}^{n} E\left(x_{i}\right) .
$$

The summation extends over the $n$ internal degrees of freedom. $n$ for benzene is thirty.

The addition of Eqs. (6) and (7) yields the heat capacity of the crystal at constant volume. To convert this into the experimentally measurable heat capacity at constant pressure, $C_{p}$, use is made of the standard thermodynamic formula

$$
C_{p}-C_{v}=\alpha^{2} V T / \beta,
$$

where $\alpha$ is the crystal's coefficient of thermal expansion, $\beta$ the coefficient of compressibility and $V$ the molar volume of the crystal. Eq. (8) expresses the contribution made to $C_{p}$ by the work required to expand the lattice.

The right-hand member of (8) can be changed to a more useful form by means of $\mathrm{Mie}^{6} \mathrm{~s}^{6}$ relation:

$$
\alpha V / \beta=\text { const. } \times C_{v} .
$$

\footnotetext{
- Mie, Ann. d. Physik 11, 657 (1903).
}

Grüneisen $^{7}$ has shown, by a combination of thermodynamic argument with the results of lattice theory, that such a relation should hold for atomic lattices whose $C_{v}$ is given by Eq. (1). Extension of .Grüneisen's reasoning to molecular lattices leads to the expression

$$
\alpha V / \beta=\sum_{i} a_{i}\left(C_{v}\right)_{i},
$$

in which the $a_{i}$ 's are constants multiplying the several components of $C_{v}$, namely $C_{v}$ due to compressional and to torsional lattice vibrations, and due to the various internal degrees of molecular freedom. Eq. (10) may be simplified by making two reasonable assumptions about the coefficients $a_{i}$. In the first place we assume, in keeping with our discussion of the relative magnitudes of the $\nu_{D}$ 's of compression and of torsion, that the coefficient of $C_{v}$ due to compressional oscillations is the same as that for $C_{v}$ due to torsional oscillations. Secondly, we suppose that the coefficients for the various components of the internal $C_{v}$ may be lumped together into one coefficient for the whole internal $C_{v}$. Thus we reduce $(10)$ to

$$
\alpha V / \beta=a^{\prime} C_{v} \text { (lattice) }+b^{\prime} C_{v} \text { (internal). }
$$

$a^{\prime}$ and $b^{\prime}$ are constants. If we make the empirically justifiable ${ }^{8}$ assumption that the quotient $V / \beta$ is temperature-independent, combination of (8) and (11) gives

$$
\begin{aligned}
& C_{p}-C_{v}=\left[a \cdot C_{v}\right. \text { (lattice) } \\
&\left.+b \cdot C_{v} \text { (internal) }\right]^{2} T .
\end{aligned}
$$

The evaluation of the constants $a$ and $b$ is essential for our calculation of $C_{p}$, but unfortunately there is at present no independent way of determining them. We should expect the constant $a$ to have a value of the order of magnitude of similar constants for atomic lattices, but no precedent exists for estimating $b$. We therefore resort to an empirical procedure for finding $a$ and $b$.

The difference between observed $C_{p}$ and calculated $C_{v}$ above $50^{\circ} \mathrm{K}$ is ascribed to work of expansion. With the help of this difference at

\footnotetext{
${ }^{7}$ Grüneisen, Handbuch der Physik, Vol. X, p. 22 ff. See also Eucken, Handbuch der Experimentalphysik, Vol. VIII, part 1, p. 281.

${ }^{8}$ Cf. Eucken, reference 7, p. 282; cf. Grüneisen, Ann. d. Physik 26, 211 (1908).
} 
any two temperatures, we can calculate $a$ and $b$ from Eq. (12). When such a calculation is carried out, ${ }^{9} a$ and $b$ are found to have the same value within about 5 percent. We may therefore set $a=b$, and reduce Eq. (12) to:

$$
C_{p}-C_{v}=k C_{v}^{2} T \text {. }
$$

TABLE I. Heat capacity of benzene and benzene- $d_{6}$.

\begin{tabular}{|c|c|c|c|c|c|c|c|}
\hline $\begin{array}{l}\text { TEM- } \\
\text { PERA- } \\
\text { TURE } \\
{ }^{\circ} \mathbf{K}\end{array}$ & $\begin{array}{l}\text { LAT- } \\
\text { TICE } \\
C_{v}\end{array}$ & $\begin{array}{c}\text { INTER- } \\
\text { NAL } \\
C_{v}\end{array}$ & $C_{p}-C_{v}$ & $\begin{array}{c}\text { CALC. } \\
C_{p}\end{array}$ & $\begin{array}{c}\text { OB- } \\
\text { SERVED } \\
C_{p}\end{array}$ & $\begin{array}{c}C_{\text {ALCC. }} \\
C_{p} \\
\left(n_{B}=160\right. \\
\left.\mathrm{cm}^{-1}\right)\end{array}$ & $\begin{array}{l}\text { CALC. } \\
C_{p} \\
C_{6} D_{B}\end{array}$ \\
\hline 4 & 0.018 & & & 0.018 & 0.0195 & .018 & .024 \\
\hline 6 & .060 & & & .060 & .065 & .060 & .074 \\
\hline 8 & .144 & & & .144 & .147 & .144 & .173 \\
\hline 10 & .270 & & & .270 & .346 & .270 & .336 \\
\hline 15 & .908 & & & .908 & .920 & .91 & 1.10 \\
\hline 20 & 1.932 & & & 1.932 & 1.84 & 1.94 & 2.29 \\
\hline 25 & 3.18 & & 0.02 & 3.20 & 3.00 & 3.24 & 3.66 \\
\hline 30 & 4.41 & & .04 & 4.45 & 4.24 & 4.57 & 4.94 \\
\hline 40 & 6.45 & & .11 & 6.56 & 6.47 & 7.06 & 7.04 \\
\hline 50 & 7.90 & & .20 & 8.10 & 8.14 & 9.20 & 8.56 \\
\hline 60 & 8.90 & 0.02 & .31 & 9.23 & 9.32 & 10.98 & 9.72 \\
\hline 70 & 9.60 & .07 & .42 & 10.09 & 10.16 & 12.48 & 10.62 \\
\hline 80 & 10.07 & .17 & .54 & 10.78 & 10.85 & 13.69 & 11.47 \\
\hline 90 & 10.42 & .31 & .67 & 11.40 & 11.44 & 14.73 & 12.29 \\
\hline 100 & 10.70 & .51 & .81 & 12.02 & 11.99 & 15.67 & 13.12 \\
\hline 110 & 10.89 & .79 & .97 & 12.65 & 12.59 & 16.50 & 13.94 \\
\hline 120 & 11.02 & 1.09 & 1.13 & 13.24 & 13.24 & 17.21 & 14.82 \\
\hline 130 & 11.14 & 1.45 & 1.33 & 13.92 & 13.96 & 17.96 & 15.75 \\
\hline 140 & 11.24 & 1.84 & 1.55 & 14.63 & 14.71 & 18.66 & 16.73 \\
\hline 150 & 11.32 & 2.31 & 1.80 & 15.43 & 15.49 & 19.46 & 17.78 \\
\hline 160 & 11.38 & 2.77 & 2.07 & 16.22 & 16.26 & 20.19 & 18.91 \\
\hline 170 & 11.43 & 3.26 & 2.37 & 17.06 & 17.10 & 20.97 & 20.13 \\
\hline 180 & 11.48 & 3.80 & 2.71 & 17.99 & 18.02 & 21.80 & 21.39 \\
\hline 190 & 11.53 & 4.31 & 3.08 & 18.92 & 19.00 & 22.62 & 22.76 \\
\hline 200 & 11.58 & 4.95 & 3.53 & 20.06 & 20.02 & 23.66 & 24.17 \\
\hline 210 & 11.62 & 5.53 & 3.98 & 21.13 & 21.11 & 24.68 & 25.65 \\
\hline 220 & 11.65 & 6.16 & 4.50 & 22.31 & 22.24 & 25.80 & 27.20 \\
\hline 230 & 11.68 & 6.80 & 5.07 & 23.55 & 23.45 & 26.88 & 28.85 \\
\hline 240 & 11.70 & 7.47 & 5.69 & 24.86 & 24.75 & 28.11 & 30.51 \\
\hline 250 & 11.72 & 8.13 & 6.35 & 26.20 & 26.09 & 29.38 & 32.28 \\
\hline 260 & 11.73 & 8.77 & 7.05 & 27.55 & 27.75 & 30.67 & 34.12 \\
\hline 270 & 11.74 & 9.46 & 7.83 & 29.03 & 29.70 & 32.09 & 35.98 \\
\hline
\end{tabular}

9 The numerical determination of the two constants is made most reliably by utilizing the $C_{p}-C_{v}$ difference at two rather widely different temperatures, e.g. at $150^{\circ}$ and $250^{\circ}$. The constants found at these two temperatures may be used in turn to calculate the expansion term for all other temperatures. The constants may also be evaluated separately. In the region $60^{\circ}-100^{\circ}, C_{v}$ (internal) makes no appreciable contribution to the $C_{p}-C_{v}$ difference. Hence the constant $a$ may be determined independently of $b$ at any temperature in this region. The evaluation of $b$ is then possible at a single temperature above $100^{\circ}$, preferably at a temperature high enough so that the $\dot{C}_{p}$ (internal) term is relatively large. The $a$ and $b$ values found in this way agree well with those found by simultaneous solution of two numerical equations obtained from Eq. (12). It is of interest to note that the value of the constant $a, 8.3 \times 10^{-3}$ reciprocal calories, agrees reasonably well with the value one would compute from the formula proposed by Nernst and Lindemann (Zeits. f. Elektrochem. 17, 817 (1911)) for this constant, namely $a^{2}=0.0214 / T_{m}$, where $T_{m}$ is the fusion temperature of the crystal. The value of $a$ calculated from this expression is $8.77 \times 10^{-3}$.
The constant $k\left(k \cong a^{2} \cong b^{2}\right)$ has the value of $6.45 \times 10^{-5}$ reciprocal calories for crystalline benzene.

\section{Calculation of $C_{p}$ for Benzene}

With the help of Eqs. (6), (7) and (13) we have calculated $C_{p}$ for crystalline benzene over its entire temperature range. The value of $\theta$ used in (6), namely $\theta=150$, was obtained in the manner we have described. (See the discussion of Eqs. (3)-(6).) The internal frequencies for Eq. (7) have been taken from our previous paper. ${ }^{1}$ The results of the calculation are listed in Table I together with values obtained by interpolating at a regular series of temperatures between the experimental results of Ahlberg, Blanchard and Lundberg ${ }^{3}$ and Nernst ${ }^{10}$ below $90^{\circ} \mathrm{K}$, and of Huffman, Parks and Daniels ${ }^{11}$ above $90^{\circ}$.

It will be seen from Table I that the agreement between calculation and experiment is remarkably good except for several points in the neighborhood of $25^{\circ} \mathrm{K}$. These rather small discrepancies are probably to be ascribed to some fluctuation in the value of $\theta$. Such fluctuations have been discussed by Blackman. ${ }^{4}$ He points out that $\theta$ should fluctuate somewhat at low temperatures but should tend to remain constant at higher values of $T$. (For "higher values" one might say "values of $T$ larger than $\theta / 2$." Compare, for example, Blackman's Fig. 3, page 375, for one-dimensional lattices.)

For our purposes it is sufficient if $\theta$ remains reasonably near to 150 in the range above $80^{\circ} \mathrm{K}$, for in that range the lattice $C_{v}$ has very nearly attained its classical value and is not very sensitive to moderate changes in $\theta$. The temperature region above $80^{\circ}$ is of primary interest because in this region the internal vibrations make their contribution to $C_{p}$. It will be noticed that from $50^{\circ}$ to $250^{\circ}$ the agreement of theory with observation is quite satisfactory. This close check may be regarded as an excellent confirmation of the values of the internal frequencies used in Eq. (7). The slight divergence of theory and experiment close to the melting point may be due on the one hand to inaccuracy of Eq. (6)

${ }^{10}$ W. Nernst, Ann. d. Physik 36, 395 (1911).

${ }^{11}$ Huffman, Parks and Daniels, J. Am. Chem. Soc. 52, $1547(1930)$. 


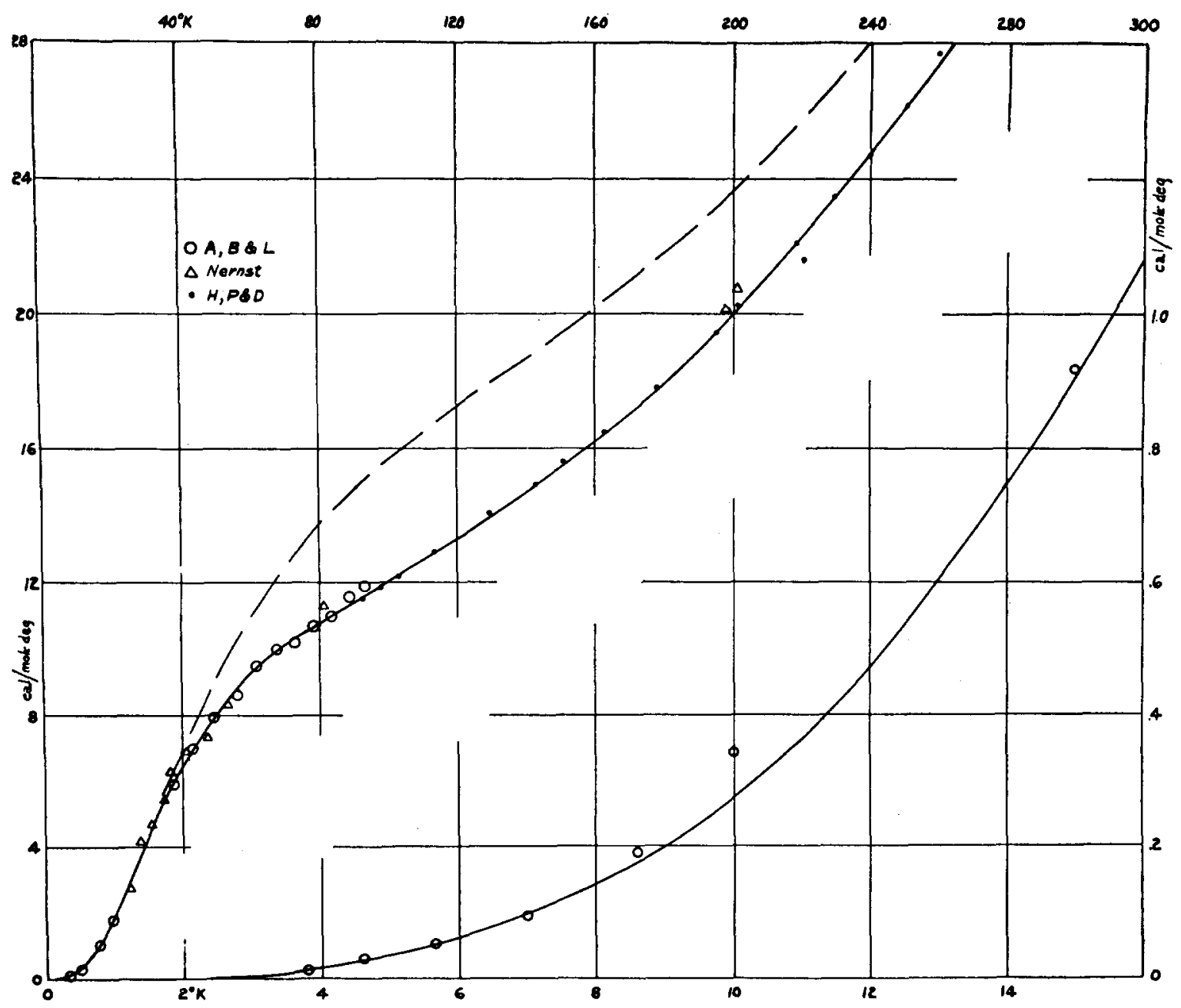

FIg. 1. The calculated values of the heat capacity of benzene with $\nu_{16}=406 \mathrm{~cm}^{-1}$ and $\nu_{4}=538 \mathrm{~cm}^{-1}$ (solid line); with $\nu_{16}=160 \mathrm{~cm}^{-1}$ and $\nu_{4}=212 \mathrm{~cm}^{-1}$ (dashed line). The experimental observations are taken from Ahlberg, Blanchard and Lundberg, ${ }^{3}$ Nernst, ${ }^{10}$ and Huffman, Parks and Daniels. ${ }^{11}$

or (13) when fusion is imminent, or on the other to slight increase in observed $C_{p}$ near the melting point because of the presence of a trace of impurity in the sample under observation.

The exactness with which the theoretical and experimental $C_{p}$ 's coincide indicates that fundamental frequencies below $400 \mathrm{~cm}^{-1}$ can hardly be present in benzene. Evidence from the band spectra of benzene, however, has often been interpreted as demonstrating the presence of a fundamental frequency of $160 \mathrm{~cm}^{-1}$. If such a fundamental were to exist in benzene, it would perforce belong to vibrational modes 4 or 16 (Wilson's notation ${ }^{12}$ ). The reasons for this assign-

${ }_{12}$ Wilson, Phys. Rev. 45, 706 (1934). ment have been previously discussed. ${ }^{1,13}$ If we assume that $\nu_{16}$ has a value of $160 \mathrm{~cm}^{-1}$, then $\nu_{4}$ must lie somewhere in the neighborhood of 212 $\mathrm{cm}^{-1}$. Column 7, Table I, gives $C_{p}$ for benzene calculated on the assumption that $\nu_{16}$ and $\nu_{4}$ have the respective values $160 \mathrm{~cm}^{-1}$ and 212 $\mathrm{cm}^{-1}$, rather than $406 \mathrm{~cm}^{-1}$ and $538 \mathrm{~cm}^{-1}$. The latter values (see reference 1) were used in obtaining the values of "calculated $C_{p}$ " in the fifth column. The $C_{v}$ portion of the values in column 7 has been obtained as before from Eqs. (6) and (7). To find the $C_{p}-C_{v}$ term, we have used Eq. (13), in which the constant $k$ has

${ }^{13}$ Angus, Bailey, Hale, Ingold, Leckie, Raisin, Thompson and Wilson, J. Chem. Soc. (London) 984 (1936). 
been assigned the empirically determined value $6.45 \times 10^{-5} \mathrm{cal}^{-1} .^{14}$ For comparative purposes these results have been plotted in Fig. 1. It will be seen from this plot that a fundamental frequency of $160 \mathrm{~cm}^{-1}$ is incompatible with the heat capacity curve over the whole range $50^{\circ}-250^{\circ} \mathrm{K}$.

\section{Prediction of $C_{p}$ For Benzene- $d_{6}$}

A calculation of the heat capacity of benzene- $d_{6}$ seemed desirable because comparison of theoretical and experimental $C_{p}$ 's should indicate just how accurate are the low internal frequencies which have been worked out for that molecule.

The lattice $C_{v}$ for benzene- $d_{6}$ is assumed to be given by Eq. (6). The shift in $\theta$ was estimated by presuming that

$$
\begin{aligned}
& \theta_{1}{ }^{\prime}=\theta_{1}\left(M / M^{\prime}\right)^{\frac{1}{2}}, \\
& \theta_{2}{ }^{\prime}=\theta_{2}\left(I / I^{\prime}\right)^{\frac{1}{2}},
\end{aligned}
$$

where $\theta_{1}$ and $\theta_{2}$ are the compressional and torsional $\theta$ 's, respectively (see Eq. (5)). $M$ is the molecular weight and $I$ any moment of inertia of benzene. The primes refer to the corresponding quantities in benzene- $d_{6}$. (There is no ambiguity in regard to the moments of inertia, for the ratio $I / I^{\prime}$ is the same for all three moments in benzene.) From (14) and the assumption that $\theta_{1} \cong \theta_{2}$, we find that

$$
\theta^{\prime}=(\theta / 2)\left[\left(M / M^{\prime}\right)^{\frac{1}{2}}+\left(I / I^{\prime}\right)^{\frac{1}{2}}\right] .
$$

(15) yields the value $\theta^{\prime}=140$.

The vibrational frequencies of benzene- $d_{6}$ as given in reference 1 were used in Eq. (7) to

${ }^{14}$ One might object, with considerable reason, that it is hardly legitimate to use here a constant found empirically under the assumption of different values for certain of the internal frequencies. This objection may be answered in part by remarking that the value of the constant used is close to that given by the independent formula of Nernst and Lindemann (reference 9). In addition it should be remembered that we are attaching no particular theoretical significance to the hypothetical $C_{p}$ curve other than its utility in demonstrating the improbability of the presence of the very low frequencies in benzene. Even if the expansion term is disregarded entirely, the low frequencies still cause some contradiction with experiment, for in the region $80^{\circ}-140^{\circ} \mathrm{K}$ the calculated $C_{v}$ is larger than observed $C_{p}$ by 1-2 percent. compute the internal $C_{v}$. The constant $k$ in Eq. (13) was taken as $6.45 \times 10^{-5}$ reciprocal calories. Column 8 , Table I, gives $C_{p}$ for benzene- $d_{6}$.

Despite the rough nature of the method of finding $\theta^{\prime}$, it is likely that our estimate of the $\theta-\theta^{\prime}$ shift is fairly good, so that the contribution of the lattice $C_{v}$ to the net specific heat has been pretty closely estimated. Presumably the expansion formula is as valid as it proved to be for benzene. The accuracy of our prediction, then, depends upon the accuracy with which the internal frequencies are known. Excepting $\nu_{4}$ and $\nu_{16}$, the important (i.e., low) frequencies in benzene- $d_{6}$ have been observed spectroscopically and are known with a precision adequate for our purposes. The calculation of $C_{p}$ therefore hinges to a large extent on the correctness of our estimate of $\nu_{4}$ and $\nu_{16}$.

The assignment of $\nu_{16}$ in benzene to the faint Raman frequency at $406 \mathrm{~cm}^{-1}$ is apparently substantiated by the excellence of agreement between theoretical and observed $C_{p}$ 's. It is to be remarked, however, the doubt here lies not in the value of the Raman line in question but in the assignment of $\nu_{16}$ to it. If the assignment is correct, we know the value of $\nu_{16}$ to within two or three $\mathrm{cm}^{-1}$. Unfortunately, the corresponding line has not been observed for benzene$d_{6}$, so that it is necessary to calculate the frequency at $\nu_{16}$ in the latter. There is some considerable degree of doubt about the accuracy of such a calculation and therefore we can scarcely expect to find the same excellence of agreement between theoretical and experimental $C_{p}$ 's for benzene- $d_{6}$ as was obtained for benzene. The measurement of $C_{p}$ for crystalline benzene- $d_{6}$, which will be undertaken in the immediate future, is awaited therefore with considerable interest, for if such measurement agrees closely with our calculation, the frequencies of the distortion vibrations 4 and 16 have been correctly estimated. The extent of any discrepancy between theory and observation, on the other hand, will indicate the size and direction of the error in our estimate of $\nu_{4}$ and $\nu_{16}$. 